UCRL-ID-120004

\title{
LDRD Final Report
}

\author{
R. E. Stewart, D. Price, R. Shepherd, W. White, R. Walling, \\ R. More
}

March 16, 1995

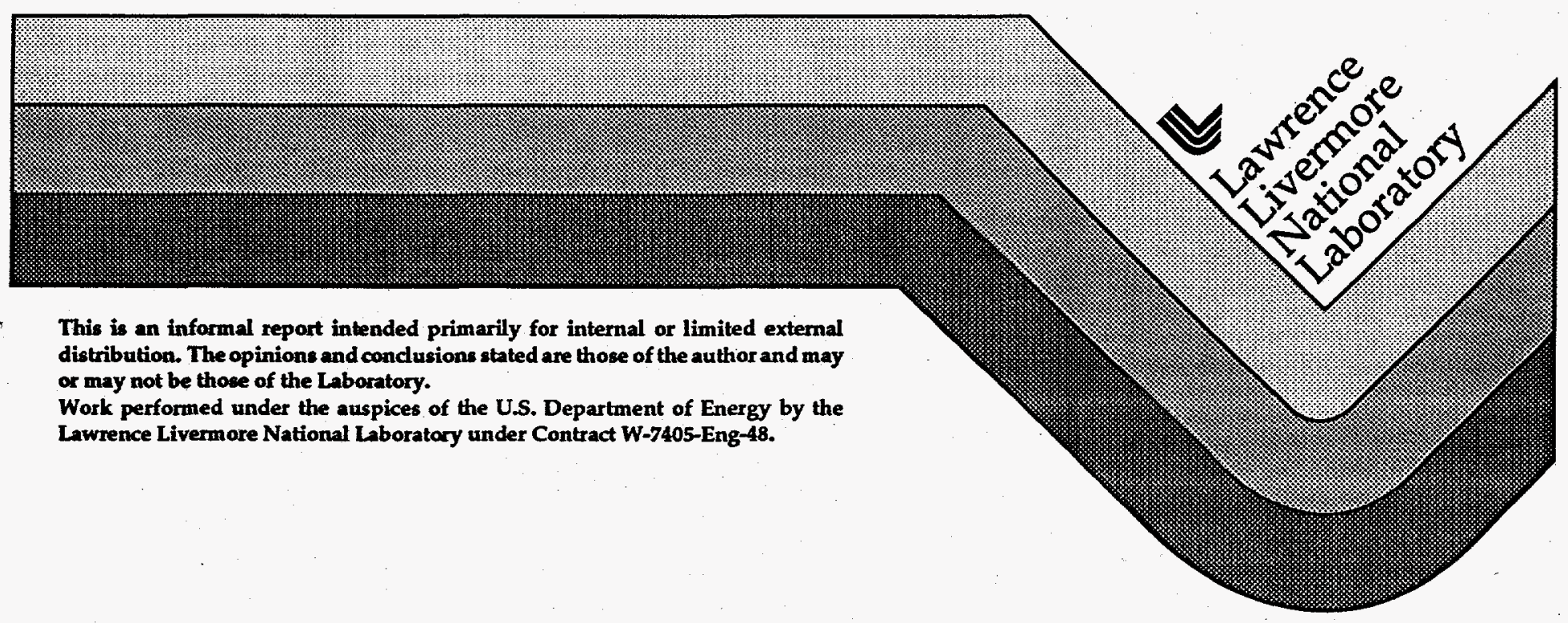




\section{DISCLAIMER}

This report was prepared as an account of work sponsored by an agency of the United States Government. Neither the United States Government nor any agency thereof, nor any of their employees, make any warranty, express or implied, or assumes any legal liability or responsibility for the accuracy, completeness, or usefulness of any information, apparatus, product, or process disclosed, or represents that its use would not infringe privately owned rights. Reference herein to any specific commercial product, process, or service by trade name, trademark, manufacturer, or otherwise does not necessarily constitute or imply its endorsement, recommendation, or favoring by the United States Government or any agency thereof. The views and opinions of authors expressed herein do not necessarily state or reflect those of the United States Government or any agency thereof. 


\section{DISCLAIMER}

Portions of this document may be illegible in electronic image products. Images are produced from the best available original document. 


\title{
LDRD Final Report
}

\author{
Principal Investigator: R.E. Stewart \\ Co-Investigators: Dwight Price, Ronnie Shepherd, William White, \\ Rosemary Walling, Richard More \\ Responsible AD: Richard Fortner - NTES \\ LDRD Tracking Code 93-SR-068
}

The goal of this project is to develop a 100 -fs pulse length laser capable of heating solid density plasmas to near-kilovolt temperatures before hydrodynamic decompression of the target can take place, and to experimentally determine the properties of these plasmas with it. We have successfully developed the laser for this work and measured plasma production and laser absorption with it. This work has demonstrated the capacity to produce solid-density plasmas. Future experiments are described. 


\section{$1 \quad$ Highlights for 1993}

This year we successfully increased the output of our Ti:Sapphire laser with the addition and testing of a flashlamp pumped dye converted amplifier. We have achieved 0.250 joule pulses with 100 femtosecond pulse widths at a repetition rate of $10 \mathrm{~Hz}$. By focusing the beam with an off-axis parabolic mirror, a 1.8 times diffraction-limited spot on target of approximately 5 micron (FWHM) at a peak power of $6.6 \times 10^{18} \mathrm{~W} / \mathrm{cm}^{2}$ has been produced. We have begun the construction of a small Nd Silicate glass rod pumped Ti: Sapphire disk to achieve $2.0-\mathrm{J}, 100$-fs power levels later this year. This should achieved focused intensities in excess of $5 \times 10^{19} \mathrm{~W} / \mathrm{cm}^{2}$. In addition, we have begun fabrication of test components for a 100-TW 10-20 Joule upgrade of the Ti:Sapphire laser system. This is currently scheduled for completion at the end of 1995 .

At these high intensities, small scale wings or prepulses in the beam can produce low density plasmas at the interface of a solid target and a vacuum that will lead to target decompression. The major source of poor contrast on our present pulses has been traced to uncorrected 3rd and 4th order phase terms in the pulse stretcher due to dispersion in the glass components of our system. We have designed a new stretcher which will fully correct this problem and reduce prepulses and wings in the compressed pulse to insignificant levels. This design was published in Optics Letters $[1,2]$ and patented this year, and construction of the system was begun. In addition, we successfully demonstrated $50 \%$ conversion of a $50-\mathrm{mJ}$ beam to $400-\mathrm{nm}$ which has much lower prepulse levels than the fundamental beam.

Experimentally, we have demonstrated that $400-\mathrm{nm}$, prepulse free light dramatically reduced hard $x$-rays production and $3 / 2 \omega$ and $2 \omega$ emission that accompanies the formation of preformed low density plasmas in our targets. With these clean pulses we observed $20 \%$ absorption at $1 \times 10^{18} \mathrm{~W} / \mathrm{cm}^{2}$ intensities. This was far beyond the levels expected from inverse brehmsstrahlung absorption. Later experiments gave fractional absorption of approximately $8 \%$ for laser 
intensities of $10^{17}-10^{18} \mathrm{~W} / \mathrm{cm}^{2}$ and fractional scattering measurements of $2 \%$. This low scattering signal is consistent with the absence of preformed plasma and indicates that the lower absorption measurement is consistent with absorption from near-solid density targets. In addition to normal inverse bremsstrahlung, theories for additional absorption mechanisms important in short-scale length plamsas are being developed and may help to explain the residual difference with current LASNEX predictions which are closer to $4 \%$ fractional absorption.

From ion time-of-flight and x-ray spectroscopy measurements, we have deduced that this absorption produced $200-700 \mathrm{eV}$ surface plasmas that cooled rapidly after the laser pulse. The $x$-ray and ion spectra are qualitatively consistent with our LASNEX simulations and particle-in-cell (PIC) simulations. We will extend the results of these simulations to higher intensities in the coming year using PIC simulations. These studies indicate that the absorption mechanism will change to reflected-ion heating at about $10^{20} \mathrm{~W} / \mathrm{cm}^{2}[3]$. Our calculations indicate that this mode of absorption is very favorable for our purposes of creating high-temperature near-solid density plasmas, and heats about 10-20 microns of material over an ion range to kilovolt temperatures.

From the results achieved this year, and scaling the simulations of our present experiments, we are optimistic that solid density material can be heated to high temperatures successfully with a 100-fs laser of the type we are presently constructing.

\section{Creating High Density Plasmas}

Lasers capable of operating with hundred femtosecond pulse lengths and intensities approaching $10^{19} \mathrm{~W} / \mathrm{cm}^{2}$ have only recently become available to experimentalists. If these lasers produce sufficiently clean pulse profiles with extremely short temporal rise-times then hydrodynamic expansion of the target material prior to the arrival of the bulk of the energy can be less than the skin 
depth for absorption. This unique feature of short scale length absorption for the laser-plasma interaction enables solid-density plasma research without the added complication of an intervening low density high temperature plasma. It is now practical to study a material's laser absorption properties through the transition between a state dominated by the solid state band structure of the electronic configuration and a higher temperature regime clearly described as an ionized plasma. Prior to the advent of short pulse lasers the description of laser energy absorption into solid material was more a description of plasma energy transport between the critical surface and the solid density material far removed from direct interaction with the laser light.

A series of experiments to measure short pulse laser light absorption in solid density materials can now be done on the LLNL Ultra-Short Pulse laser facility. Total reflected, scattered, and transmitted light measurements define the net absorption for a $100-\mathrm{fs}, 400-\mathrm{nm}$ laser pulse's interaction with a wide range of solid density target materials including $\mathrm{Al}, \mathrm{Au}, \mathrm{Ta}$, and Quartz over the intensity range of $10^{13}$ to $10^{18} \mathrm{~W} / \mathrm{cm}^{2}$. This range is sufficiently broad to observe a transition in absorption from a low intensity region dominated by the cold materials electronic band structure, to an absorption at the highest intensities marked by a convergence to a nearly universal, material independent value. At the lowest intensities each material exhibits a unique reflectivity suggestive of the materials cold, undamaged reflectivity. In the intensity range of $10^{13}$ to $2 \times 10^{14} \mathrm{~W} / \mathrm{cm}^{2}$ the effects of reorganization of electronic structure caused by laser heating to temperatures of $1-50 \mathrm{eV}$ are evident. Above $10^{16} \mathrm{~W} / \mathrm{cm}^{2}$ the reflectivity of all materials converges to a universal plasma mirror behavior. The use of frequency doubling is essential to provide a pulse contrast sufficiently clean to enable solid density absorption studies above $5 \times 10^{16} \mathrm{~W} / \mathrm{cm}^{2}$.

Besides the interest in short pulse laser absorption in solid targets as a measure of the material energy loading and thus achievable temperature, absorptive studies can provide measurements of AC electrical properties of material un- 
der conditions of extreme energy loading. Prior studies of material reflectivity to determine electrical properties of plasmas include the shock release studied of Celliers and $\mathrm{Ng}[4]$ and the 450-fs reflectivity techniques of Milchberg et al. [5], the use of shorter pulses and angle dependence by Fedosejevs et al.[6] and Downer et al.[7], was performed at lower intensities. Our work extends the above work by providing measurements over a broad range of intensities with direct comparison of a wide selection of target material. The use of a shorter pulse length than reported in most previous work and improved prepulse control by harmonic conversion combine to provide much cleaner measurements of the targets absorption with minimum hydrodynamic expansion.

\section{The LLNL 100-Femtosecond Laser Facility}

The Ti:Sapphire laser can deliver 0.1 Joule energy on target at $800 \mathrm{~nm}$ with a pulse width between 150 and $100 \mathrm{~nm}$ at a $10-\mathrm{Hz}$ repetition rate. Peak intensities on target are. $5 \times 10^{18} \mathrm{~W} / \mathrm{cm}^{2}$. Near bandwidth limited (9-nm. FWHM at 800 $\mathrm{nm}$ ), short pulses are generated in a commercially available mode locked $\mathrm{CW}$ pumped Ti:Sapphire oscillator. The pulses extracted in a $82-\mathrm{MHz}$ train are stretched to $400 \mathrm{ps}$ in a single grating diffraction grating stretcher. One 4 nanojoule chirped pulse out of the $82-\mathrm{MHz}$ train is electo-optically selected to seed the cavity of the regenerative amplifier by polarization rotation. After approximately 12 passes in the regenerative amplifier the pulse is ejected by a second firing of the Pockels cells.

Due to residual birefringence and imperfect polarized reflectivity in the thin film polarizer a small amount of energy leaks out of the regenerative cavity during each of the 12 round trips, Thus producing a series of prepulses separated by $12 \mathrm{~ns}$ (the cavity round trip time). The prepulse to main pulse intensity contrast at the regenerative amplifier is measured to be $10^{-4}$. The addition of a Pockels cell with gland polarizers between the regenerative amplifier and 
the double pass amplifier improves the contrast to $10^{-7}$. After amplification in the double pass amplifier the pulse is passed thought a second Pockels cellgland prism combination to further improve the contrast to better than $10^{-9}$. The final amplification stage is a flash lamp pumped-dye converted Ti:Sapphire amplifier.

The resultant beam is recompressed to 100 -fs in a parallel grating compressor with a reflectivity loss of $50 \%$. The grating compressor is housed in vacuum box to avoid self phase modulation and B-integral modulation of the beam. Transmittive optics are minimized to avoid B-integral and self phase modulation of the beam and generally consist of the $0.15-\mathrm{cm}$ doubling crystal operated at $41 \%$ efficient and two $0.1-\mathrm{cm}$ beam splitters and a $5-\mu \mathrm{m}$ thick nitrocellulose debris shield. The pulses are focused on target by reflection of an off-axis parabolic mirror of focal length $10: 2 \mathrm{~cm}$ operated at an f-number of 4.8 .

A short scale length plasma is required for these measurements in near-solid density plasmas. This necessitates that the laser pulse be as clean as possible. Sources of prepulse such as long pulse mode lasing of the regenerative amplifier and leakage out of the regenerative amplifier have been measured and minimized. It is known that $3 \mathrm{rd}$ and 4 th order aberrations in the pulse stretcher design introduce low level temporal wings in the pulse. A correction for these aberrations will be incorporated in the near future by adoption of a phase adjustable stretcher design[1, 2]. At intensities greater than $5 \times 10^{15} \mathrm{~W} / \mathrm{cm}^{2}$, the output from the laser is not sufficiently free of prepulse to prevent the formation of significant performed plasma prior to the arrival of the peak of the pulse. Frequency doubling is therefore employed to improve the pulse contrast sufficiently that the prepulse issues are negligible. A high dynamic range autocorrelation of the $1 \mathrm{w}$ signal implies that the $2 \omega$ beam's temporal profile is approximately:

$$
I_{2 \omega}(t)=I_{o}\left[e^{-\left(\frac{\ell}{75}\right)^{2}}+0.075 \times e^{-\left(\frac{\ell}{180}\right)^{2}}+0.00005 \times e^{-\left(\frac{t}{367}\right)^{2}}\right]
$$




\section{Measurement of Laser Absorption}

The target region is enclosed in a light integrating half cylinder of Lambertian scattering material in a functional approximation to an Ulbricht sphere. The scattered light from mirror like targets has been previously verified to be Lambertian. The scattered light is measured by a photodiode viewing a portion of the interior surface of the sphere but blocked from viewing the direct emission from the target. The incident energy and reflected energies are monitored on each shot on a pyroelectric energy meter off a calibrated beam splitter in the light path. A third pyroelectric meter monitors the directly transmitted beam through the target. All energy meters are calibrated relative to the transmission meter and transmission calibrations are made before and after each intensity scan of 200 shots so that a linear correction for debris build up on the debris shield can be made (approximately $3 \%$ change). The scattered light calibration is made by moving a calibrated near Lambertian scatterer into the defocused beam on each target run to provide a known scatter efficiency. Spectral measurements indicate that the spectral shifts in scattered and reflected light are sufficiently small to be negligible compared to the accuracy in this measurement. No attempt is made to separate backscattered and reflected light off the target. In all cases absorption is assumed to be:

Absorption $=1$ - Scattered - Reflected - Transmission.

Intensity on target is varied by two means. By changing the focal spot size on target, or by varying the total beam energy. Due to the limited total energy available the variation of intensity by reduction in energy is limited to less than three orders of magnitude with acceptable accuracy. In the majority of runs the intensity is varied by changing the focal spot diameter by moving the target relative to best focus position. The target is moved in both directions relative to best focus to insure that the best focus has been accurately located. The intensity on target is inferred by the gaussian beam propagation formula obtained by optically measuring the focus spot diameter (FWHM) on target 
of a reduced intensity beam. A non damaging ( to the target) power level of less than $10^{11} \mathrm{~W} / \mathrm{cm}^{2}$ is used. The focal spot image is obtained by viewing a calibrated magnified image of the target surface viewed through the final focusing objective, an off-axis parabola. The validity of spot diameters obtained in this manner has been verified by knife edge scans of the full power beam.

A data run consists of taking 100 to 200 measurements over an intensity range of 5 orders of magnitude at a shot rate of 1 hertz. The target is raster scanned at a rate that insures that only clean target is hit for each shot. The targets consisting primarily of e-beam deposited thin films of 1 micron thickness, deposited on 8.3 by $10.2 \mathrm{~cm}$ Kodak projector slide cover glass. For a typical data run (see Figure 1), $2 \%$ of the incident light is observed to be scattered in a lambertian distribution about the specular angle for the "mirror like" finish metallic targets. Higher scatter efficiencies have been observed and the increase has been related to target sample preparation. At the highest intensities reflectivities approaching $90 \%$ are observed. This high intensity behavior appears to be independent of the target material for "mirror like" finish samples of $\mathrm{Al}$, $\mathrm{Ta}, \mathrm{Au}$, and Quartz (see Figure 2). For aluminum a transition knee is observed between the region where absorption is dominated by inverse bremsstrahlung from the materials valance band electrons and a region increasingly dominated by absorption in a highly ionized solid density plasma with temperatures greater than $50 \mathrm{eV}$. Future absorption studies will help to elucidate the differences in absorption for the variety of materials shown in Figure 2. Here one see enormous differences in laser absorption (opacity in the $1-50 \mathrm{eV}$ range) for simple conductors like $\mathrm{Al}$; more complex conductors like $\mathrm{Cu}$, $\mathrm{Ta}$ and $\mathrm{Au}$; and insulators like Quartz. 


\section{Theory and analysis}

Extensive numerical analysis for aluminum have been completed and will be reported elsewhere. The one dimensional Lagrangian hydrodynamics simulation in LASNEX includes solution of the Felmholz equation for the laser electromagnetic field using a comprehensive model for electrical conductivity of the target material[8]. This is extended by the Drude model to AC conductivity. Also included are equation of state with separate treatment of the electron and ion temperature[9] and uninhibited thermal conduction. For the case of absorption in aluminum the departure from NLTE is not a significant factor in the laser absorption and a simple LTE model suffices.

The calculations for aluminum (Fig. 3) show similar features to the experimental measurements and may be used as a guide to the probable conditions existing at the various intensities. For intensities below $2 \times 10^{14} \mathrm{~W} / \mathrm{cm}^{2}$ laser penetration into the Aluminum target is on the order of a skin depth, 10 nanometers. Laser energy is being absorbed in this region by inverse bremsstrahlung, equivalent to $\mathrm{AC}$ Joule heating or interband absorption by conduction electrons. There is little hydrodynamic motion or heat conduction during the 100 femtosecond pulse. The target is heated to temperatures of 1 to $50 \mathrm{eV}$ for laser intensities in the range of $10^{13}$ to $2 \times 10^{14} \mathrm{~W} / \mathrm{cm}^{2}$ with the temperature dependence of the absorption arising from changes in the electron-ion collision crosssection.

Above intensities of $2 \times 10^{14} \mathrm{~W} / \mathrm{cm}^{2}$ the calculations predict a temperature greater than $50 \mathrm{eV}$ resulting in strong ionization and hence decreasing skin depth. Also absorption is reduced due to the decrease in electron - ion coulomb scattering with increasing temperature. Heat flow into the surrounding target material is significant at the high intensities. Hydrodynamic expansion is significant during the laser pulse and produce a profile which is exponential like an isothermal rarefaction, $n(r)=n \times \exp (-x / L)$ where $L$ is the electron density scalelength, but the temperature and density are not constant in space or time. 
At the highest intensities pondermotive pressure steeping of the density profile is apparent. The absorption of aluminum above $10^{17} \mathrm{~W} / \mathrm{cm}^{2}$ experimentally is in the range of $8 \%$. This is higher than the $3-4 \%$ predicted by inverse bremsstrahlung alone. Additional absorption may include an absorption process associated with reflection of the free electrons by the plasma sheath. Simple estimates for this absorption have been made by the theory of Catto and More[10] and when included in total absorption slightly increase absorption efficiencies.

The effects of surface contamination upon the measurement and the need for improved accuracy must be addressed before it is possible to determine the elemental dependence in absorption at the highest intensities.

\section{Summary}

This project has developed a laboratory source of high energy density 100-fs pulse length laser capable of heating high energy solid density plasmas to near$\mathrm{keV}$ temperatures. Measurements of scattered light and absorbed light proved consistent with extremely clean laser pulses as required for this goal.

This year we successfully increased the output of our Ti:Sapphire laser with the addition and testing of a flashlamp pumped dye converted amplifier. We have achieved 0.250 joule pulses with 100 femtosecond pulse widths at a repetition rate of $10 \mathrm{~Hz}$. By focusing the beam with an off-axis parabolic mirror, a 1.8 times diffraction limited spot on target of approximately 5 micron (fwhm) at a peak power of $6.6 \times 10^{18} \mathrm{~W} / \mathrm{cm}^{2}$ has been produced. We have begun the construction of a small Nd Silicate glass rod pumped Ti: Sapphire disk to achieve 2.0-J, 100-fs power levels later this year. This should achieved focused intensities in excess of $5 \times 10^{19} \mathrm{~W} / \mathrm{cm}^{2}$. In addition, we have begun fabrication of test components for a 100-TW 10-20 Joule upgrade of the Ti:Sapphire laser system. This is currently scheduled for completion at the end of 1994 .

From the results achieved this year, and scaling the simulations of our present 
experiments, we are optimistic that solid densily material can be heated to higher temperatures successfully with a 100-is laser of the type we are presently constructing. 


\section{References}

[1] W. E. White, F. G. Patterson, R. L. Combs, D. F. Price, and R. L. Shepherd. Optics Letters, 18:1343-1345, 1993.

[2] A. Sullivan and W. E. White. Optics Letters, 20, 1995.

[3] J. Denavit. Phys. Rev. Lett., 69:3052, 1992.

[4] P. Celliers and A. Ng. Phys. Rev. E, 47, 1993.

[5] H. M. Milchberg, R. R. Freeman, S. C. Davey, and R. M. More. Phys. Rev. Lett., 61:2364-2367, 1988.

[6] Fedosejevs, Ottman, Siegel, Kuhnle, Szatmari, and Schafer. Applied Phys. $B, 50: 79,1990$.

[7] M. C. Downer, Forkand, and Shank. J. Opt. Soc. Am. B, 2:595, 1985.

[8] Y. T. Lee and Richard M. More. Phys. Fluids, 27:1273-1285, 1984.

[9] R. M. More, K. H. Warren, D. A. Young, and G. B. Zimmerman. Phys. Fluids, 31:3059-3078, 1988.

[10] P. J. Catto and Richard M. More. Phys. Fluids, 20:704-705, 1977. 


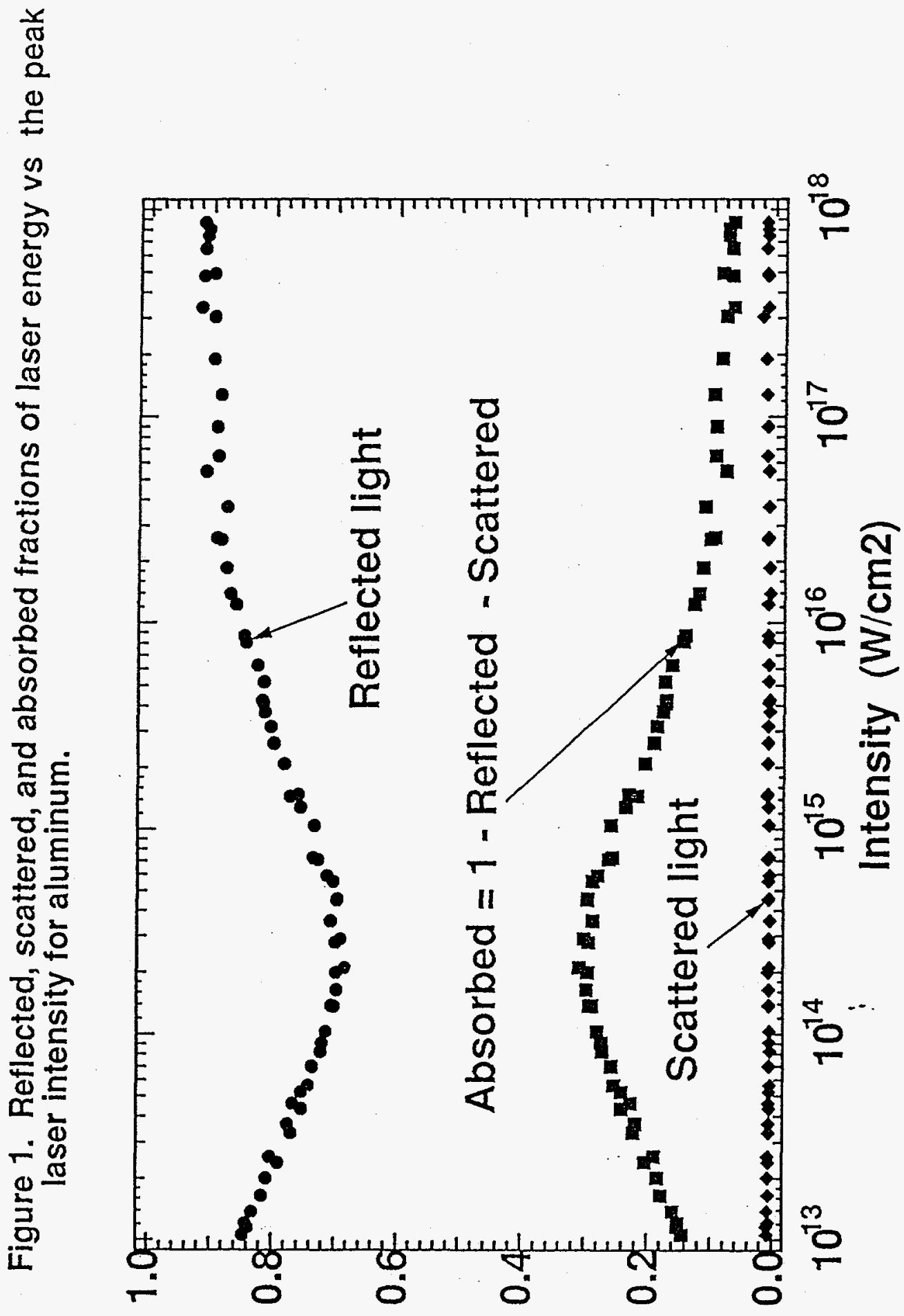


Figure 2. Experimental absorption fractions for different materials.

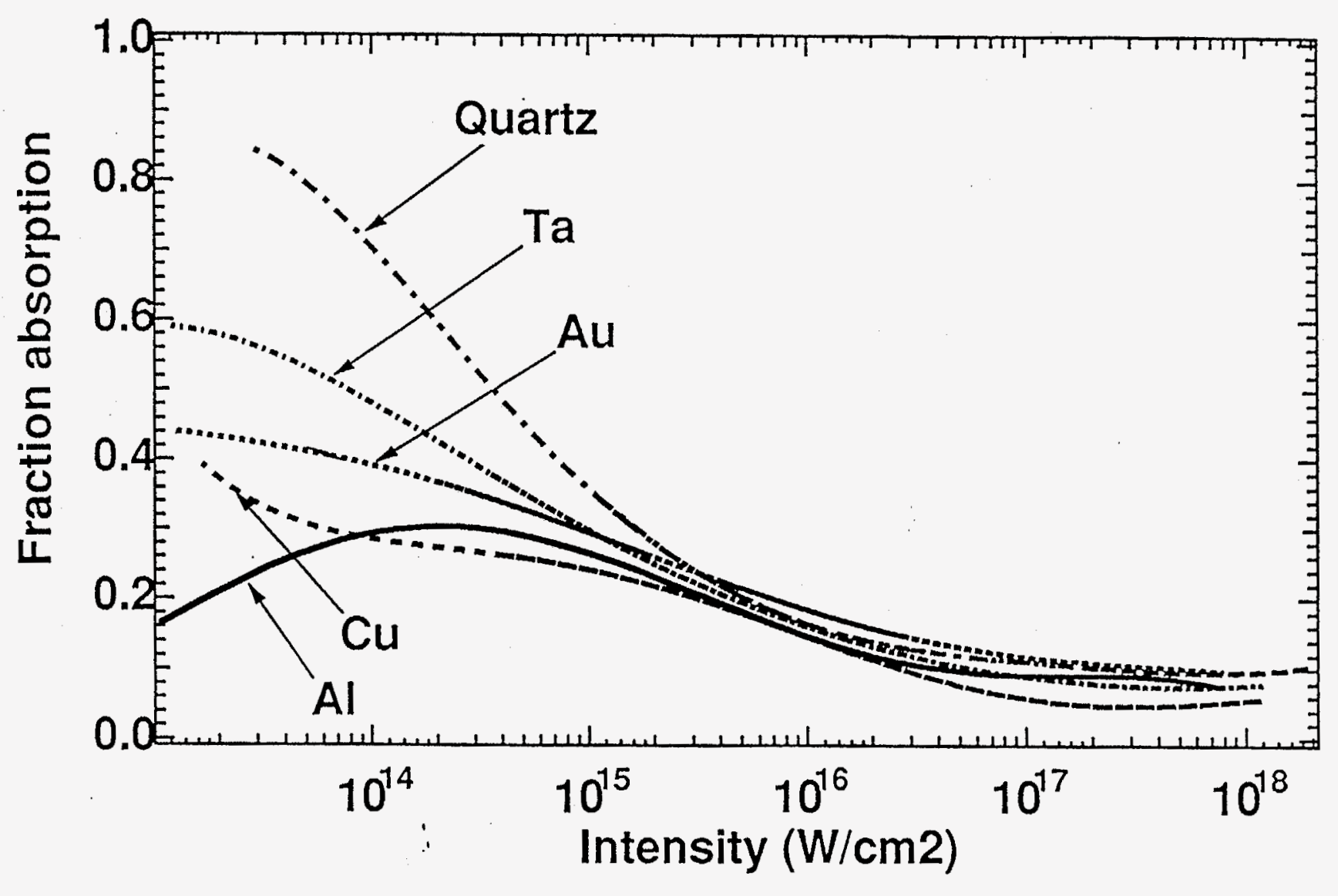


Figure 3. Squares represent the measured absorption fraction for aluminum. The solid curve gives the LASNEX prediction.

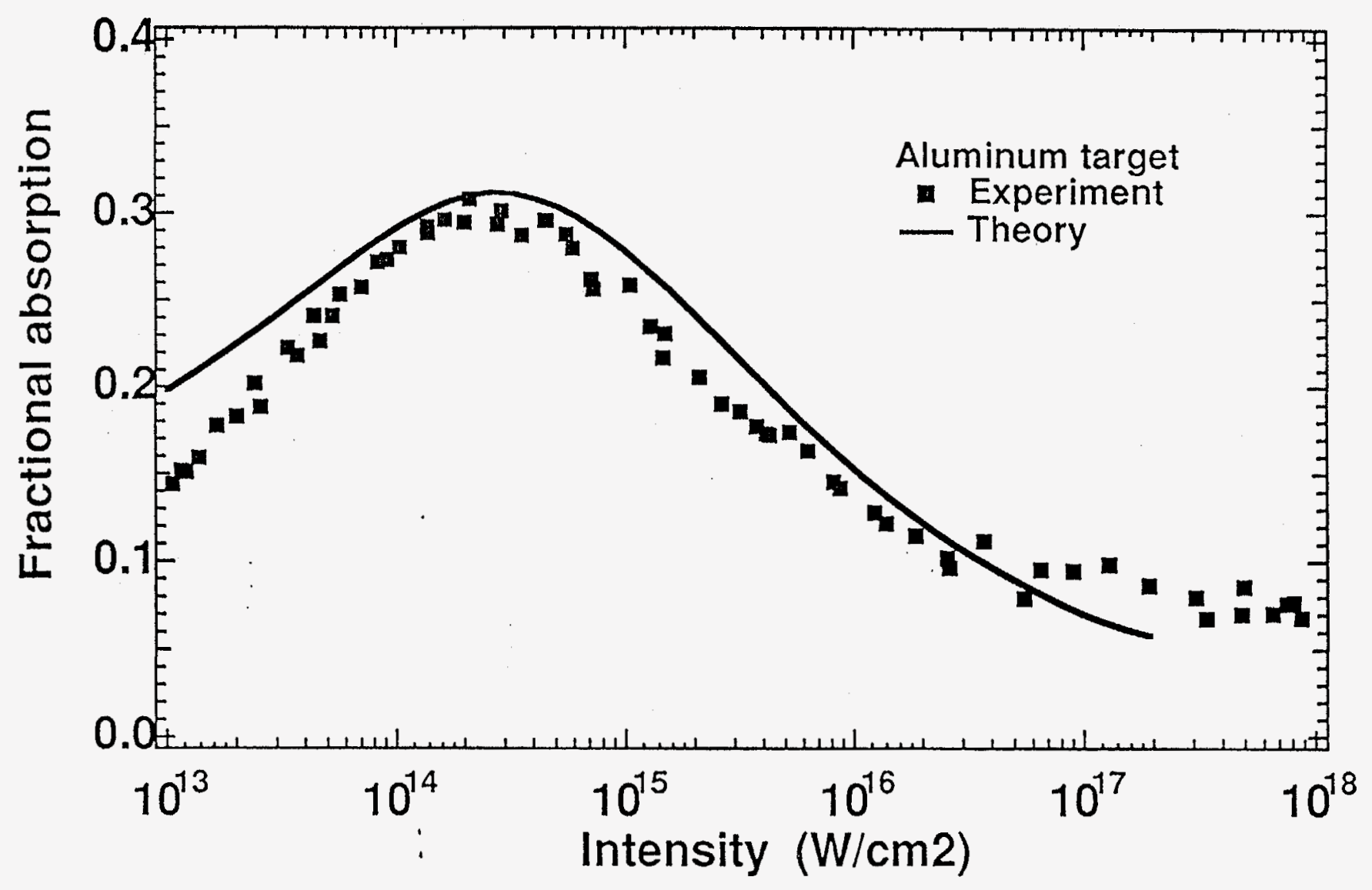

